ECCOMAS

Proceedia
COMPDYN 2021

$8^{\text {th }}$ ECCOMAS Thematic Conference on Computational Methods in Structural Dynamics and Earthquake Engineering

M. Papadrakakis, M. Fragiadakis (eds.) Streamed from Athens, Greece, 28 - 30 June 2021

\title{
SETTLEMENT-INDUCED DAMAGE ASSESSMENT IN UNILATERAL MASONRY-LIKE STRUCTURES: A PIECEWISE RIGID DISPLACEMENT APPROACH
}

\author{
Ida Mascolo ${ }^{1}$, Antonio Fortunato ${ }^{1}$ \\ ${ }^{1}$ University of Salerno \\ Department of Civil Engineering \\ Via Giovanni Paolo II, 132 - 84084 Fisciano (SA) \\ e-mail: $\{$ imascolo, a.fortunato\}@unisa.it
}

\begin{abstract}
Settlements severely affect historic masonry structures, and in fact, settlementinduced structural failures are common worldwide. This paper describes a rigid block detection and identification procedure of the crack pattern in masonry-like structures subjected to dead loads and finite displacements (e.g., settlements). The procedure is based on the Piecewise Rigid Displacements (PRD) approach. The equilibrium problem is formulated in the framework of Limit Analysis by means of a suitable variational formulation for the Boundary Value Problem (BVP) of Normal Linear Elastic No-Tension material (NENT). The equilibrium states of masonry-like structures are obtained as an optimization of Total Potential Energy (TPE). The application of the proposed method to real case studies highlights its potential in the field of mechanics and the mechanisms of fracture.
\end{abstract}

Keywords: Masonry, arch bridge, seismic behaviour, Piecewise Rigid Displacement approach. 


\section{INTRODUCTION}

Masonry arch bridges are susceptible to extensive structural damage, failure, and even collapse when subjected to exceptional loading or massive differential settlements induced by floods, ship-worm infestations of wooden foundations, scouring, and other natural or man-made events. The Scrivia River, Trigno, and Rubianello bridges in Italy (2002, 2003, 2013), the Ballynameen and Bishopsford Road bridges in England (2015, 2019), the Deba bridge in Spain (2018) are just some examples of recent spread world wide masonry bridges failures under scour-induced settlements [1]-[6] are just a few examples of recent worldwide masonry bridges failures under scour-induced settlements. These events are further proof of the urgent need for the analysis and assessment of the load-carrying capacity of masonry bridges. The number of scientific research papers dealing with this topic is impressive. The main methods of structural analysis of masonry construction are:

- FEM-based approaches, which are subdivided into three main categories depending on the representative scale: macro-modeling [7]-[9], discontinuous models [10] and micro-modeling [11]. Moving from the macro- to the micro-scale, the level of accuracy increases, but the modeling and numerical implementation challenges and the computational efforts also significantly increase;

- homogenization approaches [12, 13], in which a structural elementary cell (consisting of different components such as mortar and blocks) is modeled as an equivalent homogeneous medium (i.e., a continuum) with mechanical parameters that are derived from each of its individual components; and

- Limit Analysis-based approaches, modern approaches that support the classical methods employing thrust line theory, graphic statics, and Limit Analysis. Their origins date to the English school in the late 1600s, with the theory of the chain having firstly been proposed by Robert Hooke (1676) and used later by Christopher Wren in Saint Paul's dome (1675), by Giovanni Poleni in St. Peter's dome in Vatican (1743), and, more recently, by Antoni Gaudí i Cornet for the design of the church of Colònia Güell in Barcelona (c. 1900) [14, 15]. Their modern formulation, due to Anthony Kooharian, Jacques Heyman and Olgierd Cecil Zienkiewicz[16]-[18], is based on the No-Tension assumption. The material is modeled as a no-tension rigid or elastic material, with unilateral no-friction contact conditions [19]-[22].

Nonetheless, the issue is far from being exhausted because each of these three methods yields results only within its own bounds. There is to date no generally accepted way to assess masonry structures.

This paper focuses on analyzing and predicting crack patterns in masonry arch bridges subjected to dead loads and finite displacements. In the framework of the modern Limit Analysis-based approaches, the equilibrium problem is reformulated with a classical variational inequality form or equivalently as a constrained minimization problem of a nondifferentiable functional (i.e., the Total Potential Energy) over the set of admissible displacements [23]-[?]. The main novelty is the consideration of a factitious elasticity of the material to guarantee the convergence of the proposed method. The solution is then obtained by making the elastic deformation tensor tend to zero. This procedure is based on the Piecewise Rigid Displacements approachn [21], which consists of restricting the minimization problem by a finite class of piecewise rigid displacements with support on a 
finite partition of the whole structural domain. This reduces the equilibrium problem to a convex optimization problem for which existence and uniqueness are ensured. The case study of the Deba Bridge failure in Spain is also considered to capture the essence of the proposed method and its effectiveness.

The structure of the paper is as follows: Section 2 focuses on the Deba Bridge and its failure occurred in 2018, Section 3 presents the mathematical model and theoretical framework of the proposed numerical approach, Section 4 applies such a procedure to the case study of Deba Bridge. The results show a satisfactory agreement between the numerical-based crack pattern and the observed one, proving to be a powerful and versatile tool for the study of the mechanisms of fracture of No-Tension materials. The proposed method can be usefully exploited for the study of collapse mechanisms and ultimate load capacity of a wide range of masonry-like structures as it is emphasized in the concluding remarks drawn in Section 5.

\section{The Deba Bridge}

The Deba Bridge is a four-span stone bridge connecting the city of Deba with Mutriku in the Basque Country of Spain. It was built from 1865 and 1866 as part of a larger coastal motorways project that aimed to link the cities of San Sebastián and Bilbao. For its artistic and historic value, the Deba Bridge has special protection within the Monumental Complex of the Camino de Santiago. The underlying geology of the Deba riverbed is mainly composed of alluvial soil mixed with silt, sand, and gravel that constantly experiences landslides, dolines, and rockfalls. This is reflected in many episodes of settlement-induced bridge failures over the years. The latest occurred at the beginning of July 2018, when the central pier experienced a vertical settlement of about $0.80-0.90 \mathrm{~m}$, resulting in a great depression in the road, damage and cracking of the two central bridge vaults, and, consequently, closure of the bridge (Figure 1).

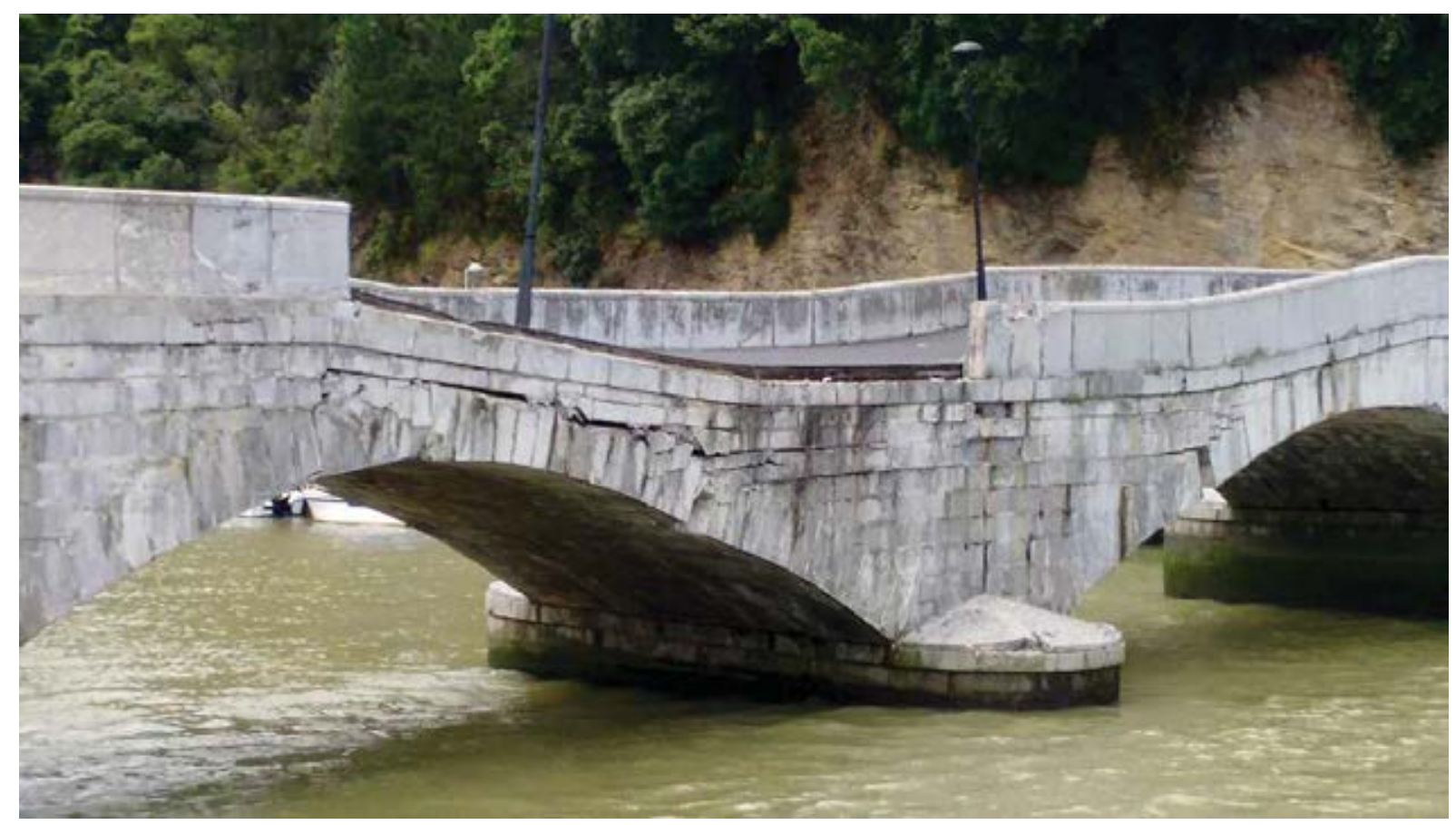

Figure 1: Views of the Deba Bridge (Debako Zubia kaltetuta) 


\section{Mathematical model}

Let us consider an elastic body that occupies, in its undeformed state, an open and bounded subset $\Omega \in \Re^{2}$ with a sufficiently smooth boundary (i.e., $\mathcal{C}^{1,1}$-regular curve $\partial \Omega$ ), consisting of two open disjoint parts $\partial \Omega=\partial \Omega_{D} \cup \partial \Omega_{N} \cup$. They are bound to firsttype (i.e., Dirichlet) and second-type (i.e., Neumann) boundary conditions (i.e., here, the displacements and the surface forces are prescribed), respectively:

$$
\mathbf{u}=\overline{\mathbf{u}} \quad \text { on } \partial \Omega_{D}, \quad \mathbf{T}^{(n)}=\mathbf{p}, \mathbf{b} \quad \text { on } \partial \Omega_{N} .
$$

Here, $\overline{\mathbf{u}}$ denotes the vector field of prescribed surface displacements; $\mathbf{p}=\left\{p_{i}\right\}$ and $\mathbf{b}=\left\{b_{i}\right\}$ denote the prescribed forces and the body forces; and $\mathbf{T}^{(n)}=\mathbf{T} \mathbf{n}$, where $\mathbf{n}$ is the outward normal vector on the boundary of $\Omega$. Let $\mathbf{E}=\boldsymbol{\varepsilon}+\boldsymbol{\lambda}$ denote the infinitesimal strain tensor, additively decomposed into an elastic $(\varepsilon)$ and an anelastic part $(\boldsymbol{\lambda})$; $\mathbf{T}$ the stress tensor; and $\mathbb{C}$, the positive-definite elasticity tensor. The constitutive equations for the NENT material we investigate are described by the set of relations:

$$
\begin{array}{ll}
\text { strain-stress relation: } & \mathbf{E}=\mathbb{C}^{-1} \mathbf{T}+\boldsymbol{\lambda}, \\
\text { internal constraint: } & \mathbf{T} \in S^{-} \mathrm{m}^{-}, \\
\text {normality law: } & \boldsymbol{\lambda} \in \mathrm{Sym}^{+} \wedge \mathbf{T} \cdot \boldsymbol{\lambda}=0 .
\end{array}
$$

The Boundary Value Problem (BVP) for a NENT material is given by:

Problem 3.1 Find an admissible state $(\mathbf{u}, \boldsymbol{\lambda}, \mathbf{T})$ (i.e., displacement, anelastic strain and stress fields):

1. equilibrium

$$
\operatorname{div} \mathbf{T}+\mathbf{b}=\mathbf{0}, \quad \text { on } \Omega
$$

2. stress-strain constitutive law

$$
\mathbf{T}=\mathbb{C}(\mathbf{E}-\boldsymbol{\lambda})
$$

3. internal constraint and normality law

$$
\mathbf{T} \in \text { Sym }^{-}, \boldsymbol{\lambda} \in \text { Sym }^{+}, \mathbf{T} \cdot \boldsymbol{\lambda}=0,
$$

4. boundary conditions

$$
\mathbf{T n}=\mathbf{p}, \mathbf{u}=\left.\overline{\mathbf{u}}\right|_{\partial \Omega_{N}}, \quad \text { on } \partial \Omega_{N} .
$$

The resulting weak formulation, i.e., the variational formulation of such a problem, can be formulated as follows:

Problem 3.2

$$
\begin{aligned}
\operatorname{minimize}_{\mathbf{u} \in \mathcal{K}, \varepsilon}: & \mathcal{E}_{p}(\boldsymbol{\varepsilon})=\int_{\Omega} \phi_{\mathbb{E} i}(\varepsilon) \mathrm{d} a-\int_{\Omega} \mathbf{b} \cdot \mathbf{u} \mathrm{d} a-\int_{\partial \Omega_{N}} \mathbf{p} \cdot \mathbf{u} \mathrm{d} s, \\
\text { subject to }: & \mathbf{A U} \geq 0, \quad \mathbf{B U}=0
\end{aligned}
$$

where $\mathcal{K}$ is a proper finite class of piece-wise rigid displacements obeying material conditions and having support on a finite partition of the whole domain $\Omega=\bigcup_{1}^{m} \Omega_{i}$

$$
\mathcal{K} \subset\left\{\mathbf{u} \in \mathcal{S}(\Omega): \boldsymbol{\lambda} \in \operatorname{Sym}^{+} \text {and } \mathbf{u}=\overline{\mathbf{u}} \text { on } \partial \Omega_{N}\right\}
$$


$\phi_{\mathbb{E} i}$ denotes the strain energy density, $\mathbf{U} \in \Re^{3 m}$ is the vector collecting the three Lagrangian parameters of the elementary mesh element $\Omega_{i}$, and the matrices $\mathbf{A} \mathbf{U}$ and $\mathbf{B U}$ collect the no-concentration and no-sliding conditions, respectively (See ... for more details). Note that the resulting optimization problem involves a quadratic functional subjected to linear constraints. By minimizing the TPE with respect to both displacements and elastic deformation, we are forcing the elastic part of the strain tensor to vanish. It is equivalent to coupling the initial rigid structure, characterized by a positive semi-definite stiffness $\mathbf{K}$, with a fictitious elastic structure, characterized by a positive-definite stiffness $\mathbf{K}_{s}$. It can be proven that the problem of finding an admissible state of displacement for the initial structure is equivalent to the same problem formulated for a structure having a positive-definite stiffness, $\mathbf{K}_{\delta}=\mathbf{K}+\delta \mathbf{K}_{s}$, because regularization parameter $\delta$ tends to zero [30].

\section{Numerical analysis}

The proposed method is validated by analyzing the case study of the Deba Bridge failure. The convex optimization problem related with the proposed approach was solved here by the Interior Point method [31,32], which is particularly advantageous when the problem involves a large number of variables $(3 \cdot 1012$ in the case analyzed here). The bridge domain is discretized into 1012 quadrilateral elements and a vertical displacement of $0.9 \mathrm{~m}$ and a counterclockwise rotation of 1.13 is imposed on the central pier of the bridge. - The obtained results, both in terms of displacements and macro-block identification, show good agreement with the failure geometrical survey. On the basis of blocks rigid roto-translations, six macroblocks have been identified. The two on the outside (the light blue blocks in Figure 2) are fixed. Then a four-block isostatic substructure can be identified, the kinematic motion of which is closer to that seen in the bridge failure (Figure 2).

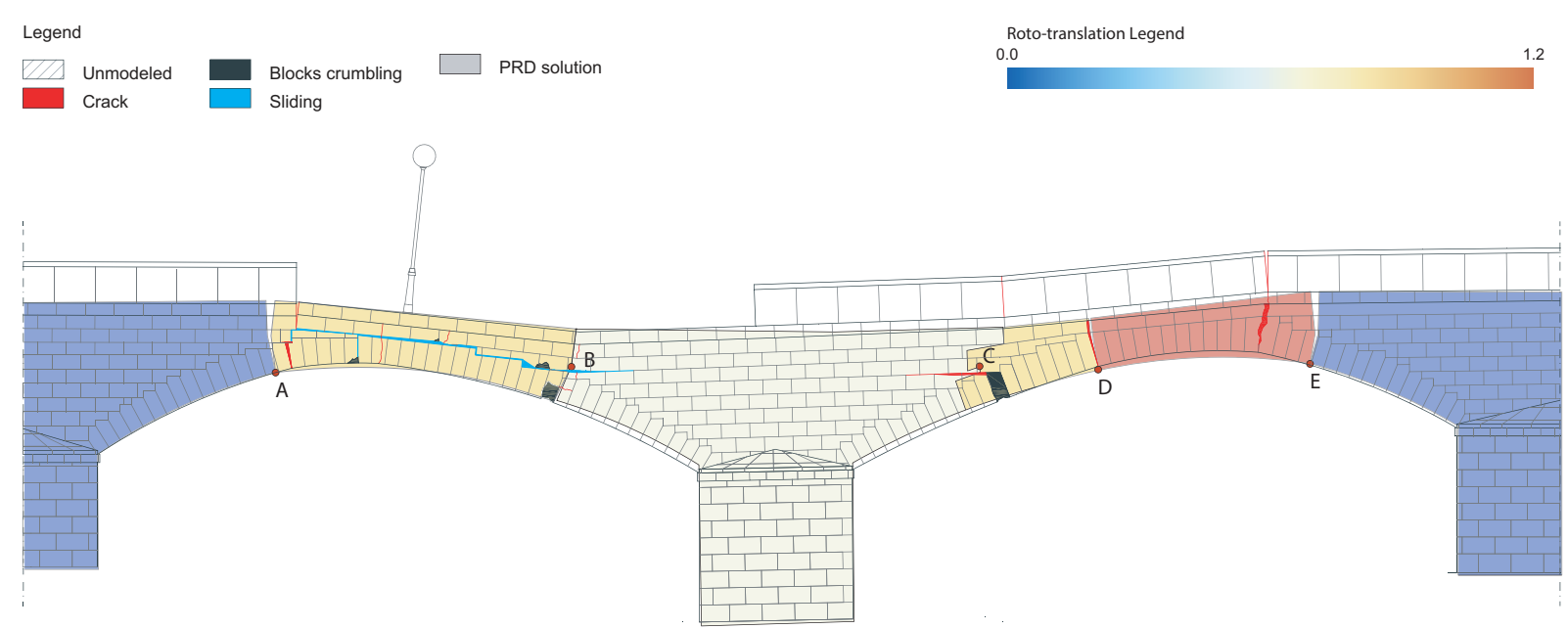

Figure 2: Superposition of PRD numerical results and failure geometrical survey (red dots A, B, C, D, and E mark the hinges location between two adjacent macroblocks).

\section{CONCLUSIONS}

A preliminary study of the mechanics of fracture mechanisms in unilateral no-tension bridge structures subjected to pier settlements is proposed here. This approach falls into 
the so-called block-based Limit Analysis methods. The equilibrium problem is formulated by a convex optimization problem with a quadratic objective function and linear inequality constraints. The main innovation introduced by this approach is consideration of a Normal Linear Elastic No-Tension material in which the elastic part of the strain tensor is considered only fictitious. By minimizing the TPE with respect to both displacements and elastic deformation, we force the elastic part of the strain tensor to vanish. In this way, the problem of finding an admissible state of displacement for the NENT structure became equivalent to the same problem formulated for a No-Tension No Rigid material. The proposed approach is applied to a case study of the Deba Bridge failure (2018). The obtained results indicate very good agreement between the numerical model and the failure survey. The method is simple, effective, and promising. The range of applicability of this method can be broadened to facade and complex NENT structures. It provides a good starting point for the study of the collapse mechanisms and ultimate load capacity of masonry-like structures.

\section{REFERENCES}

[1] Zampieri P., Zanini, M.A. Faleschini, F. Hofer L., Pellegrino C., 'Failure analysis of masonry arch bridges subject to local pier scour', Eng. Fail Anal., Vol. 79, pp. $371-84,2017$.

[2] Tubaldi E., Macorini L., Izzuddin B.A., 'Three-dimensional mesoscale modelling of multi-span masonry arch bridges subjected to scour', Eng. Struct., Vol. 165, pp. 486-500, 2018.

[3] S. Galassi, G. Misseri, L. Rovero, G. Tempesta, Failure modes prediction of masonry voussoir arches on moving supports Engineering Structures 173 (2018) 706-717

[4] Scozzese F., Ragni, L. Tubaldi E. and Gara F., Modal properties variation and collapse assessment of masonry arch bridges under scour action, Engineering Structures, Vol. 199, 109665, 2019.

[5] A. Tralli, A. Chiozzi, N. Grillanda, G. Milani, Masonry structures in the presence of foundation settlements and unilateral contact problems. International Journal of Solids and Structures, 191-192, 187-201, 2020.

[6] Malena M., Angelillo M., Fortunato A., de Felice G., Mascolo I., Arch bridges subject to pier settlements: continuous vs. piecewise rigid displacements methods, Meccanica, 2021. (Under Review)

[7] Benedetti A., Pelà L., Aprile A., Masonry properties determination via splitting tests on cores with a rotated mortar layer., A: International Seminar on Structural Masonry. "8th International seminar on structural masonry: proceedings: 05-07 November 2008". Estambul: Instanbul Technical University, 1-8, 2008.

[8] Mallardo V., Malvezzi R., Milani E., Milani G., Seismic vulnerability of historical masonry buildings: A case study in Ferrara, Engineering Structures, 30(8), 22232241, 2008. 
[9] Pelà L., Aprile A., Benedetti A., Seismic assessment of masonry arch bridges, Engineering Structures, 31(8), 1777-1788, 2009.

[10] Pegon P., Pinto A.V., Géradin M., Numerical modelling of stone-block monumental structures, Computers \& Structures, 79(22-25), 2165-2181, 2013.

[11] Casolo S., Macroscale modelling of micro-structure evolution damage by a rigid body and spring model, J. Mech. Mater. Struct., 4(3), 551-570, 2009.

[12] Zucchini A., Lourenco P.B., A micro-mechanical model for the homogenisation of masonry, International Journal of Solids and Structures, 39(12), 3233-3255, 2002.

[13] Milani G., Lourenco P.B., Tralli A., Homogenised limit analysis of masonry walls, Part I: Failure surfaces,, IComputers Structures, 84(3-4), 166-180, 2006.

[14] Rafols, J.F., Gaudì 1928, Universitat Politecnica de Cataluya. Iniciativa Digital Politecnica; N. 1 edición (4 julio 2011), 2021.

[15] Heyman J., Hooke's cubico-parabolical conoid, Notes Rec. R. Soc., 52(1), 39-50, 1989.

[16] Kooharian A., Limit Analysis of voussoir and concrete arches, J. Amer. Concrete Inst., 24, 317-328, 2021.

[17] Heyman J., Limit Analysis of voussoir and concrete arches, Int. J. Solids and Structures, 2, 249-279, 1966.

[18] Zienkiewic O.C., Valliappan S., King I.P., Stress analysis of rock as a 'No Tension' material, Géotechnique, 18(1), 56-66, 1968.

[19] Lucchesi M., Padovani C., Pasquinelli G. and Zani N., 'Masonry constructions: mechanical models and numerical applications', Lecture Notes in Applied and Computational Mechanics, 39, Springer, Berlin, 2008.

[20] Angelillo M., Fortunato A., Gesualdo A., Iannuzzo A., Zuccaro G. 'Rigid block models for masonry structures', International Journal of Masonry Research and Innovation, 3(4), 349-368, 2018.

[21] Iannuzzo A., Olivieri C., Fortunato A., Displacement capacity of masonry structures under horizontal actions via prd method. Journal of Mechanics of Materials and Structures, 14(5), 703-718, 2019.

[22] Angelillo M., Olivieri C., DeJong M.J., A new equilibrium solution for masonry spiral stairs. Engineering Structures, 238, 112176, 2021. DOI: 10.1016/j.engstruct.2021.112176

[23] Anzellotti G. A class of convex non-coercive functionals and masonry-like materials, Ann. Inst. Henri Poincaré, 2(4), 261-307, 1985.

[24] Del Piero G., Constitutive equation and compatibility of the external loads for linear elastic masonry-like materials, Meccanica, 24(3), 150-162, 1989. 
[25] Giaquinta M., Giusti E., 'Researches on the equilibrium of masonry structures', Arch. Ration. Mech. An., 88(4), 359-392, 1985.

[26] Mascolo I., Fulgione M., Pasquino M., Lateral torsional buckling of compressed open thin walled beams: experimental confirmations. Int. J. Masonry Research and Innovation, 4(1/2), 150-158, 2019. DOI: 10.1504/IJMRI.2019.096829

[27] Olivieri C., Angelillo M., Gesualdo A., Iannuzzo A. and Fortunato A., Parametric design of purely compressed shells. Mechanics of Materials, 155, 103782, 2020.

[28] Fortunato A., Gesualdo A. and Mascolo I. and Monaco M., P-Bézier energy optimization for elastic solutions of masonry-like panels. Int. J. of Masonry Research and Innovation, 2021. (In Press) DOI: 10.1504/IJMRI.2021.10037508

[29] Mascolo I., Gesualdo A., Olivieri C., Fortunato A., On rigid blocks detection in unilateral masonry-like structures. Int. J. of Masonry Research and Innovation, 2021. (In Print)

[30] Stavroulakis G.E., Panagiotopoulos P.D., Al-Fahed A.M., On the rigid body displacements and rotations in unilateral contact problems and applications. Computers \& Structures, 40(3), 599-614, 1991.

[31] Boyd S., Vandenberghe L., Convex Optimization. Cambridge University Press, 2004.

[32] Dantzig G.B., Orden A., Wolfe P., The generalized simplex method for minimizing a linear form under linear inequality constraint. Pacific. J. Math., 5(2), 183-195, 2015. 